\title{
Deutschland auf dem Weg in die „Rentner-Demokratie“? - Eine empirische Untersuchung altersspezifischer Einstellungsunterschiede und ihrer Bedeutung für das Wahlverhalten auf Basis einer aktuellen Bevölkerungsumfrage ${ }^{1}$
}

\author{
Michael Bergmann / Laura Konzelmann / Hans Rattinger
}

Is Germany on the Path to a "Pensioner Democracy"? - An Empirical Investigation of AgeSpecific Differences in Political Attitudes and their Relevance for Electoral Behavior Based on a Recent Survey

Abstract: Demographic change is one of the biggest challenges with regard to social and economic developments in Germany. The public debate mainly focuses on negative effects for social security systems. However, analyzing potential results of an ageing population for political attitudes and political behavior of the citizenry does not play a major role in scientific research so far. Based on a recent survey this article first examines age-specific differences in individual political attitudes. Second, we analyze possible determinants of age-specific party vote choice. We find age-specific attitudes, which - at least in part - do have an impact on party choice.

Keywords: Demographic change, Pensioner democracy, Age-specific differences in attitudes, Vote choice, Intergenerational relationships

Schlagwörter: Demografischer Wandel, Rentner-Demokratie, altersspezifische Einstellungsunterschiede, Wahlverhalten, intergenerationelle Beziehungen

\section{Einleitung}

Der demografische Wandel ist eine der zentralen gesellschaftlichen Herausforderungen des 21. Jahrhunderts. Während er in den westlichen Industrienationen einen Bevölkerungsrückgang bei gleichzeitiger Alterung bewirkt, führt er in anderen Teilen der Welt zu einem enormen Bevölkerungswachstum. Verantwortlich für Veränderungen von Populationen hinsichtlich ihrer altersstrukturellen Zusammensetzung und ihrer Größe ist dabei das Zusammenspiel der drei die Bevölkerungsentwicklung determinierenden Faktoren. Diese sind die Fertilitätsrate, die durchschnittliche Lebenserwartung sowie die Migrationsrate.

1 Die Daten wurden im Rahmen des von der VolkswagenStiftung geförderten Projekts „Auswirkungen des demographischen Wandels auf politische Einstellungen und politisches Verhalten in Deutschland“ erhoben, das an der Universität Mannheim durchgeführt wird. 
In Deutschland liegt die durchschnittliche Kinderzahl pro Frau bereits seit den 1970er-Jahren konstant bei ungefähr 1,4 und unterschreitet somit deutlich den Wert von 2,1, der den Erhalt der Population sichern würde. Parallel dazu steigt die durchschnittliche Lebenserwartung seit vielen Jahrzehnten kontinuierlich an - eine Änderung dieses Trends ist nicht in Sicht (vgl. Bundesministerium des Innern 2011, S. 13-18, 21-25). Das Zusammenwirken dieser Entwicklungen führt im Ergebnis dazu, dass der Alterungs- und Schrumpfungsprozess im internationalen Vergleich in Deutschland stark vorangeschritten ist. Es besteht weitgehend Konsens darüber, dass diese Entwicklung nicht aufzuhalten ist, sondern allenfalls abgemildert werden kann. Folgende Eckdaten verdeutlichen die massiven Änderungen in der altersstrukturellen Zusammensetzung der deutschen Bevölkerung: Seit 1970 ist der Bevölkerungsanteil der unter 20-jährigen von 30 Prozent auf unter 19 Prozent im Jahr 2010 gesunken. Im gleichen Zeitraum stieg der Anteil der Personen über 59 Jahren von 19 auf etwa 26 Prozent an. Dieser Trend wird auch in Zukunft Bestand haben und könnte, der aktuellen Bevölkerungsprognose des Statistischen Bundesamtes zufolge, zu einer Halbierung des Anteils der Jungen im Jahr 2060, verglichen mit dem Niveau im Jahr 1970, führen, der dann bei nur noch etwas mehr als 15 Prozent liegen würde. Gleichzeitig könnte sich der Anteil der über 59-jährigen im Vergleich zu 1970 mehr als verdoppeln und somit auf über 40 Prozent steigen. ${ }^{2}$

Zumindest ein Teil der deutschen Bevölkerung dürfte seit längerem um die voranschreitende gesellschaftliche Alterung wissen - insbesondere weil die damit verbundenen Konsequenzen für die Sozialversicherungssysteme seit einigen Jahren vermehrt von den Medien aufgegriffen werden. ${ }^{3}$ So ist beispielsweise absehbar, dass die Zahl der Erwerbstätigen in den nächsten Jahren zurückgehen wird, gleichzeitig aber eine wachsende Zahl von Rentnern finanziert werden muss. Zudem werden die Kosten im Gesundheits- und Pflegesektor sowie der Bedarf an Pflegekräften in die Höhe schnellen. Hier sind unter anderem Maßnahmen notwendig, welche die Selbständigkeit der Menschen bis ins hohe Alter zu erhalten helfen. Gleichzeitig benötigt die sinkende Zahl Jüngerer Unterstützung. Diesbezüglich sind Förderprogramme im Bildungssektor unabdingbar, die jedem Kind, unabhängig vom sozialen Status seines Elternhauses, reelle Chancen auf ein erfolgreiches Erwerbsleben eröffnen. In der Familienpolitik wiederum sind Vorschläge gefragt, die dazu beitragen, jungen Paaren die Realisierung ihres Kinderwunsches zu erleichtern.

Diese Auflistung von erforderlichen Maßnahmen zum bestmöglichen Umgang mit einer schrumpfenden und alternden Bevölkerung ist sicher nicht vollständig,

2 Die Bevölkerungsanteile der Altersgruppen beruhen auf Daten des Statistischen Bundesamtes, die unter www.destatis.de am 08.03.2012 abgerufen wurden. Die Prognosen entstammen Szenario W1-1 der 12. Koordinierten Bevölkerungsvorausberechnung, das auf folgenden Annahmen beruht: Einer annähernd konstanten Geburtenziffer (1,4 Kinder pro Frau), einer leicht steigenden Lebenserwartung bis 2060 (männlich: 85,0 Jahre, weiblich: 89,2 Jahre) und einem jährlichen positiven Wanderungssaldo von 100.000 Personen ab 2014.

3 Empirische Befunde aus mehreren europäischen Ländern zeigen, dass Befragte durchaus um die Gefahren der gesellschaftlichen Alterung für die sozialen Sicherungssysteme wissen und den Anteil Älterer eher zu hoch als zu niedrig einschätzen (vgl. Dorbritz 2008, S. 31). 
verdeutlicht aber den wachsenden, nicht weiter zu ignorierenden Problemdruck. Dass es auf den genannten und weiteren Gebieten - wie etwa dem Arbeitsmarkt - umfassenden Reformbedarf gibt, wird mittlerweile auch von den politisch Verantwortlichen erkannt. ${ }^{4}$ In den Wahlprogrammen zur letzten Bundestagswahl ist die gesellschaftliche Alterung ein von allen im Bundestag vertretenen Parteien aufgegriffenes Thema. Erste Maßnahmen wurden mittlerweile ergriffen, wie beispielsweise die Einführung der schrittweisen Verlängerung der Lebensarbeitszeit zeigt. Doch nach wie vor fehlt eine ganzheitliche Strategie, die über vereinzelte Reformen in ausgewählten Politikbereichen hinausgeht. Darüber kann auch die kürzlich vom Bundeskabinett verabschiedete „Demografiestrategie“ (Bundesministerium des Innern 2012) nicht hinwegtäuschen, die eher eine kleinteilige Bestandsaufnahme bereits existierender Programme und Vorhaben darstellt als ein konsequentes Angehen der genannten, seit langem bekannten Problematik (vgl. Klingholz 2012).

Ein Grund für das Fehlen einer solchen „echten“ Strategie ist sicherlich, dass demografische Verschiebungen nur langsam vonstatten gehen und nicht eines Tages urplötzlich sichtbar werden. Dieser Aspekt der gesellschaftlichen Alterung verhindert, das Thema mit einer gewissen Dringlichkeit anzugehen. Darüber hinaus ist ein weiterer Aspekt von Bedeutung: Parteien riskieren nur ungern den Verlust von Wählerstimmen, den umfassende Reformen der Sozialversicherungssysteme nach sich ziehen würden. Insbesondere im Vorfeld von Wahlen erwecken Parteien bisweilen den Eindruck, als ob sie den vermeintlichen politischen Ansprüchen der älteren Bevölkerung in besonderem Maße entgegen kommen, um diese als Wähler nicht zu verprellen. Auch die Autoren des sechsten Altenberichts der Bundesregierung beschreiben diese Haltung, die den Älteren indirekt mehr Macht verleihe, welche darin bestehe, „[...] dass Politiker und Politikerinnen Entscheidungen treffen, von denen angenommen wird, sie würden von älteren Menschen positiv bewertet (und deshalb mit den Wählerstimmen der Älteren honoriert)“ (Deutscher Bundestag 2010, S. 237). Eine Politik, die in erster Linie ihre Wiederwahl im Auge hat, verhält sich insofern durchaus rational, wenn sie lediglich kurzzeitig Luft verschaffende Korrekturen vornimmt, auf objektiv notwendige, starke Einschnitte jedoch verzichtet beziehungsweise diese auf zukünftige Legislaturperioden verschiebt.

Zusammenfassend ist festzustellen, dass die öffentliche, aber auch die wissenschaftliche Diskussion über die Folgen der Alterung vor allem um die Finanzierbarkeit der Sozialversicherungssysteme und benachbarte Bereiche wie die Versorgung der älteren Bevölkerung oder die Förderung von Familien und Kindern kreist. Im Zusammenhang mit dem wachsenden Anteil der älteren Bevölkerung stellt sich vor diesem Hintergrund die Frage, wie ausgeprägt deren Reformbereitschaft in politischen Bereichen ist, von denen weniger sie selbst als die Jüngeren profitieren. Gleichzeitig besteht die Befürchtung, dass die Repräsentation der Interessen der jüngeren Bevölkerung aufgrund des Übergewichts älterer Wähler nicht mehr ausreichend gewährleistet sein könnte. Der Grund dafür wird in der überproportional hohen Wahlteilnahme Älterer und deren höheren Präferenz für die

4 Wenngleich eingewendet werden kann, dass dies seit geraumer Zeit bekannt ist (vgl. etwa Miegel 1981). 
Unionsparteien gesehen (vgl. Goerres 2010, S. 111-112). Wissenschaftliche Arbeiten, die sich diesem Thema der Wahlforschung widmen, konzentrieren sich daher größtenteils darauf, ob der vielzitierte Generationenkonflikt im Wahlverhalten bereits existent sei (vgl. u. a. Falter u. Gehring 1998) und inwiefern „grey majorities" (Goerres 2008) in der Lage seien, politische Willensbildungsprozesse und Wahlausgänge in einem Regierungssystem, dem eine Entwicklung zur „RentnerDemokratie" 5 bereits nachgesagt wurde, in ihrem Sinne zu beeinflussen. Andere Arbeiten legen den Schwerpunkt auf die Erwartungen an den Sozialstaat und untersuchen, ob es diesbezüglich altersspezifische Einstellungen gibt, die miteinander unvereinbar erscheinen (vgl. u. a. Rinne u. Wagner 1995; Dallinger 2002; Blome et al. 2008, S. 315-336).

Vor diesem Hintergrund werden wir im Folgenden altersspezifische Einstellungsunterschiede insbesondere im Hinblick auf ihre parteipolitischen Konsequenzen untersuchen. Dafür greifen wir auf aktuelle Daten einer Bevölkerungsbefragung zurück, die sich schwerpunktmäßig mit politischen Einstellungen und Verhaltensweisen im Zusammenhang mit der gesellschaftlichen Alterung beschäftigt. Unsere Analysen beleuchten zwei primär mit den Bedürfnissen Jüngerer und zwei eher mit den Bedürfnissen Älterer in Verbindung stehende politische Handlungsfelder: Die Familien- und Bildungspolitik auf der einen Seite und die Bereiche der finanziellen Absicherung und der Pflege im Alter auf der anderen Seite. Uns interessiert dabei, wie die Parteien von Jungen und Alten in diesen Politikbereichen eingeschätzt werden. Wählen Jüngere vor allem Parteien, die sie in den eher ,jungen “ Politikfeldern als kompetent erachten? Und bedeutet dies, dass angesichts der Zunahme des Anteils Älterer diejenigen Parteien, die sich in der Wahrnehmung der Bürger vor allem für „alte“ Interessen einsetzen, automatisch als Wahlsieger hervorgehen? Träfe dies zu, dann spräche in der Tat viel für eine Entwicklung hin zu einer „Rentner-Demokratie“, in welcher der Problemdruck für notwendige Reformen zwar wächst, die Durchsetzbarkeit von Maßnahmen aufgrund des steigenden Anteils Älterer gleichzeitig jedoch eher ab- als zunimmt. ${ }^{6}$

Unsere Vorgehensweise gliedert sich wie folgt: Ausgehend von einem Überblick über mögliche Ursachen altersspezifischer Einstellungs- und Verhaltensdifferenzen werden zunächst individuelle Einstellungen von Jung und Alt einander gegenübergestellt. Hierbei konzentrieren wir uns auf die Salienz der Politikbereiche Bildung, Familie, finanzielle Absicherung und Pflege im Alter sowie die gewünschte Ausgabenhöhe in diesen Politikfeldern. Zudem beleuchten wir die Wahrnehmung von Problemen in Folge des demografischen Wandels und untersuchen, inwieweit Ältere und Jüngere ihre eigenen politischen Interessen durch die Parteien vertreten sehen und welcher Partei sie in den genannten Politikfeldern die größte Kompetenz bei der Lösung von Problemen zuweisen. Zusammengenommen gibt dies

5 Der ehemalige Bundespräsident Roman Herzog verwendete diesen Begriff in einem Interview mit der Bild-Zeitung am 11.04.2008: „Ich fürchte, wir sehen grade die Vorboten einer Rentner-Demokratie: Die Älteren werden immer mehr, und alle Parteien nehmen überproportional Rücksicht auf sie. Das könnte am Ende in die Richtung gehen, dass die Älteren die Jüngeren ausplündern.“

6 Aufgrund der Datenlage beschäftigen wir uns in diesem Beitrag jedoch nicht mit der Reformbereitschaft der politischen Akteure, sondern legen den Fokus auf die Einstellungen und Verhaltensmuster der Wähler. 
Aufschluss über verschiedenartige Interessenlagen, aber auch über unterschiedlich stark ausgeprägte Befürchtungen. Im Anschluss daran werden potenzielle Einflussfaktoren im Hinblick auf ein systematisch zwischen Altersgruppen divergierendes Stimmverhalten untersucht, um Auswirkungen des demografischen Wandels für die politischen Akteure abschätzen zu können.

\section{Ursachen altersspezifischer Einstellungs- und Verhaltensdifferenzen}

Zur Identifizierung möglicher Ursachen für altersspezifische Differenzen hinsichtlich politischer Einstellungen und Verhaltensweisen wird in der Literatur in der Regel auf die Position eines Wählers im Lebenszyklus (Alters- oder Lebenszykluseffekt) sowie auf seine Zugehörigkeit zu einer bestimmten Geburtskohorte (Generationen- oder Kohorteneffekt) verwiesen (vgl. statt vieler Hagenaars 1990). ${ }^{7}$ Die Abgrenzung und Untersuchung dieser beiden Aspekte durch statistische Verfahren erfordert allerdings längsschnittliche Daten. Angesichts der hier verwendeten Datenbasis, eines 2011 erhobenen Querschnitts, ist eine Separierung nicht möglich ${ }^{8}$, diese wurde bereits an anderer Stelle geleistet (vgl. Rattinger 1992; Konzelmann et al. 2012). Jedoch erlaubt die Beschäftigung mit den genannten Effekten eine zielführende Diskussion möglicher Ursachen von Verhaltens- und Einstellungsunterschieden zwischen Alt und Jung, die im vorliegenden Beitrag im Fokus der Analyse stehen.

Der zentralen Annahme des Alterseffekts zufolge beeinflusst der individuelle Alterungsprozess politische Einstellungs- und Verhaltensmuster (vgl. Blais et al. 2004). Die Ursache hierfür liegt im Durchlaufen verschiedener Lebensphasen, die mit jeweils spezifischen Interessen, Bedürfnissen und Forderungen einhergehen. Dies wirkt sich auf individuelle politische Orientierungen aus, die somit altersbedingten Schwankungen unterliegen (vgl. Kaspar u. Falter 2007, S. 118). Im Gegensatz dazu unterliegt dem Kohorteneffekt die Prämisse, dass nicht der individuelle Alterungsprozess, sondern die jugendliche Sozialisationsphase entscheidend ist für die Ausbildung spezifischer Präferenzen und Handlungsmuster: Personen, die zu einem ähnlichen Zeitpunkt geboren werden, sind ähnlichen Einflüssen in ihrer formativen Phase ausgesetzt und entwickeln demnach auch ähnliche politische Ansichten, die, einmal erworben, eine hohe lebenzyklische Stabilität aufweisen (vgl. Jennings u. Niemi 1981, S. 122; Arzheimer 2006, S. 321).

Die beschriebenen Effekte haben unterschiedliche Implikationen für die Wahlbeteiligung und die Parteiwahl von Menschen verschiedenen Alters. Unter Rückgriff auf den Alterseffekt ist zu vermuten, dass die Wahlwahrscheinlichkeit im

7 Einige Autoren unterscheiden zusätzlich einen Periodeneffekt (vgl. Rattinger 1992; Blais et al. 2004). Dieser beschreibt wahlspezifische Ereignisse, durch die alle Gesellschaftsmitglieder, unabhängig von ihrem individuellen Alter oder Geburtsjahr, gleichermaßen beeinflusst werden.

8 Die Betrachtung ausschließlich eines Zeitpunkts ermöglicht keine gesicherte Aussage darüber, ob beispielsweise ein überproportional hoher Anteil Älterer in der Unionswählerschaft darin begründet liegt, dass diese in ihrem Lebenszyklus weiter vorangeschritten sind, sie zu einem anderen Zeitpunkt geboren wurden oder ob der Erhebungszeitpunkt und dessen Spezifika entscheidend sind. Dazu kommt, dass die Effekte in der Realität zeitgleich nebeneinander auftreten und darüber hinaus miteinander verwoben sein dürften. 
Laufe des Lebens steigt und die Wahlbeteiligungsquote älterer Wahlberechtigter daher höher ausfällt als die jüngerer. ${ }^{9}$ Mit Bezug auf den Kohorteneffekt finden sich ebenfalls Hinweise auf eine höhere Wahlbeteiligung heute Älterer (Blais et al. 2004; Goerres 2010). Dabei sind verschiedene Aspekte als Ursache zu nennen wie beispielsweise ihr höheres politisches Interesse im Vergleich zu jüngeren Generationen (Blais et al. 2004), der Bedeutungsverlust bestimmter sozialer Normen infolge veränderter Wertvorstellungen vor allem bei Angehörigen jüngerer Kohorten (Arzheimer 2006; Dalton 2008) sowie der höhere Stellenwert des Wahlrechts für die ältere Generation, der sich aus dem historischen Kontext ableitet. ${ }^{10}$

Die Daten der Repräsentativen Wahlstatistik (RWS) ${ }^{11}$ unterstützen die Implikationen beider beschriebener Effekte, wonach heute Ältere eine höhere Wahlteilnahme aufweisen als Jüngere. Während die Wahlbeteiligung bei der Bundestagswahl 2009 insgesamt bei 70,8 Prozent lag, wiesen Personen unter 40 Jahren eine unterdurchschnittliche Wahlbeteiligung auf. Am wahlunfreudigsten waren Wahlberechtige in ihren Zwanzigern. Ab 40 Jahren war die Wahlbeteiligungsquote überdurchschnittlich hoch und stieg mit fortschreitendem Alter durchgängig. Am häufigsten gingen Bürger zwischen 60 und 69 Jahren an die Wahlurne (80 Prozent). Erst bei Personen, die 70 Jahre und älter waren, erfolgte ein Rückgang auf knapp 73 Prozent.

Neben der höheren Wahlbeteiligung älterer Bürger in Deutschland wird im Zusammenhang mit der Debatte um die Genese einer „Rentner-Demokratie“ ein weiterer Aspekt betont: Die Entwicklung der Altersstruktur sorge dafür, dass Ältere in Zukunft einen wahlentscheidenden Vorteil erhielten, da ihr Populationsanteil stetig wachse. Die Jüngeren, so die Erwartung, gerieten somit zusätzlich zu ihrer niedrigen Wahlbeteiligung aufgrund ihres schrumpfenden Bevölkerungsanteils in eine benachteiligte Lage, in der die Repräsentation ihrer Interessen im politischen Prozess nicht mehr ausreichend gewährleistet sein könnte (vgl. Falter u. Gehring 1998, S. 463-464; Wolf u. Kohli 1998; Wilkoszewski 2010, S. 364-365).

9 Der Hauptgrund hierfür wird darin vermutet, dass der größere Vorrat akkumulierter Lebenserfahrung, der die Auseinandersetzung mit politischen Prozessen und Inhalten umfasst, dazu führt, dass die Wahlteilnahme für Ältere mit geringerem Aufwand verbunden ist als für Jüngere. Der wiederholte Urnengang wird für Erstere somit mehr und mehr zu einer gewohnheitsmäßigen Handlung (vgl. u. a. Arzheimer 2006, S. 321). Einschränkend ist hinzuzufügen, dass die Wahlbeteiligung im sehr hohen Alter wieder sinkt. Zuvorderst ist dieser Umstand gesundheitlichen Einschränkungen geschuldet. Ein weiterer wichtiger Grund ist im Tod des (Ehe-) Partners zu sehen, auf den häufig ein sozialer Rückzug bis hin zur Isolation folgt (vgl. Falter u. Gehring 1998, S. 467-468; Goerres 2010, S. 109).

10 Für Angehörige älterer Generationen stellt die demokratische Realität nicht in dem Maße wie für jüngere Generationen eine alltägliche Selbstverständlichkeit dar. Sie dürften die Einführung freier Wahlen in gewisser Weise als Privileg empfunden haben, was dazu beitrug, das Wahlrecht als soziale Norm in wesentlich stärkerem Ausmaß als bürgerliche Pflicht zu erachten. Das bedeutet aber nicht, dass sich Jüngere generell aus der politischen Sphäre zurückziehen. In den letzten Jahren hat sich das Spektrum alternativer politischer Partizipationsformen vielmehr erweitert. Diese neuen Partizipationsformen werden insbesondere von Jüngeren favorisiert, wohingegen die ältere Bevölkerung eher konventionelle Partizipationsformen, an erster Stelle ist hier der Urnengang zu nennen, ausübt (vgl. Dalton 2008).

11 Diese Datensammlung basiert auf zufälligen Erhebungen zum Stimmverhalten in etwa vier Prozent aller Wahlkreise. Hierbei werden Wahlzettel nach Geschlecht und Altersgruppen markiert, sodass im Gegensatz zu Umfragedaten keine Verzerrungen zum Beispiel aufgrund sozialer Erwünschtheit auftreten. Die Daten sind auf der Internetpräsenz des Bundeswahlleiters öffentlich zugänglich (www.bundeswahlleiter.de). 
Für die Bundestagswahl 2009 zeigt die Repräsentative Wahlstatistik, dass bereits fast jeder dritte Wahlberechtigte 60 Jahre oder älter war. Demgegenüber war nur rund jeder sechste unter 30 Jahre alt. Dieses Verhältnis wird sich zukünftig weiter zuungunsten der Jüngeren verändern. Damit der hohe Anteil älterer Wähler im Elektorat jedoch tatsächlich zu einer selektiven Beeinflussung politischer Prozesse führt, sind Einstellungsdifferenzen zwischen Jüngeren und Älteren erforderlich. Zudem muss die Wahl bestimmter Parteien durch divergierende altersspezifische Einstellungen in der Wählerschaft beeinflusst werden. Nur wenn beides zusammenkommt, besteht die Möglichkeit, dass die skizzierte ungleiche altersspezifische Machtbasis einen systematischen - die Jungen benachteiligenden - Einfluss auf Wahlausgänge (und weitere politische Prozesse) hat und sich Deutschland tatsächlich auf dem Weg in eine „Rentner-Demokratie“ befindet.

Wenn das Stimmverhalten von Alt und Jung zur Sprache kommt, ist eine der am häufigsten artikulierten Erwartungen, dass die Wahrscheinlichkeit, konservativ zu wählen, mit dem Lebensalter steigt (kritisch hierzu: Goerres 2010, S. 116117). Der Grund hierfür liegt, der Argumentation des Alterseffekts folgend, im zunehmenden Interesse an der Aufrechterhaltung eines einmal erreichten Lebensstandards und gesellschaftlichen Status, den Ältere (eher) durch die bestandswahrende Ausrichtung der Unionsparteien gewährleistet sehen. Die Parteiwahl der Jüngeren hingegen wird verstärkt mit der Wahl der Grünen (und seit wenigen Jahren mit der Wahl der Piratenpartei) verbunden, da diese vermehrt auf Jüngere interessierende Themen der „Neuen Politik“ (Kohler 1998, S. 538) wie beispielsweise Umweltschutz, Energie- oder Netzpolitik ausgerichtet seien (vgl. Alber 1994, S. 155-157). Aufgrund der auf das individuelle Alter zurückzuführenden Änderungen von Interessen und Bedürfnissen ist somit unter Rückgriff auf den Alterseffekt zu erwarten, dass zumindest mittelfristig insbesondere die CDU/CSU von der Alterung des Elektorats profitieren wird. ${ }^{12}$

Die Zukunftsaussichten müssen allerdings anders bewertet werden, wenn nicht der Alterseffekt, sondern der Kohorteneffekt für die Parteiwahl ausschlaggebend ist. Von entscheidender Bedeutung ist dabei der parteipolitische Kontext während der politischen Sozialisation beziehungsweise der ersten Stimmabgabe der Wähler. Empirische Untersuchungen belegen, dass ältere Generationen der Geburtsjahrgänge bis circa 1948, die in der Regierungszeit der ersten drei von der CDU gestellten Bundeskanzler ihre Wahlberechtigung erlangten und somit in einer Zeit wirtschaftlicher Erfolge und politischer Stabilität aufwuchsen, häufiger für die Unionsparteien votieren, wohingegen später Geborene, geprägt durch die erste SPD-geführte Regierung, eher zur Wahl dieser beziehungsweise der Grünen neigen (vgl. Wolf u. Kohli 1998, S. 155-157; Goerres 2010, S. 114-115; Konzelmann et al. 2012). Da eine mit dem Alter verbundene Veränderung der Parteipräferenz bei Vorliegen ausschließlich eines Kohorteneffekts dezidiert nicht erwartet wird, folgt

12 Allerdings ist nicht absehbar, wie die anderen politischen Parteien auf die gesellschaftliche Alterung reagieren. Keine Partei kann es sich leisten, die Älteren nicht auch als ihre Zielgruppe zu begreifen. Womöglich werden sie ihr Profil daher der gesellschaftlichen Entwicklung anpassen. Dadurch könnten weitere Parteien als Träger konservativer Orientierungen eine Konkurrenz für die CDU/CSU darstellen und von der Alterung der Bevölkerung profitieren. 
daraus die Erwartung, dass die zukünftig Älteren zumindest auf lange Sicht nicht länger eine ausgeprägte Affinität zu den Unionsparteien aufweisen werden, sondern auch andere Parteien von der gesellschaftlichen Alterung profitieren. ${ }^{13}$

Die skizzierten theoretischen Vermutungen und empirischen Belege, denen zufolge die Älteren häufiger für die CDU/CSU votieren, werden durch die Repräsentative Wahlstatistik bestätigt: Personen, die 60 Jahre und älter sind, haben in der bundesdeutschen Wahlgeschichte durchgängig überdurchschnittlich häufig für die Unionsparteien gestimmt. Die Differenz hat sich dabei im Zeitverlauf merklich erhöht: Von 1953 bis 1987 lag der Stimmanteil der Union in dieser Altersgruppe durchschnittlich um 4,8 Prozentpunkte höher als in der Gesamtwählerschaft, 1990 bis 2009 beträgt der entsprechende Wert 8,6 Prozentpunkte. Für den gesamten Zeitraum beläuft sich die durchschnittliche Abweichung auf 6,2 Prozentpunkte. ${ }^{14}$

Die Befunde zum altersspezifischen Wahlverhalten wurden in den bisherigen Ausführungen ursächlich mit dem Alter beziehungsweise dem Geburtsjahr in Verbindung gebracht. Im Zusammenhang mit der Untersuchung von Einstellungsdeterminanten für die Wahl einer bestimmten Partei greifen wir ergänzend auf den Self-Interest-Ansatz zurück, dessen Argumentation eine auf den Maximen der rationalen Nutzenmaximierung beruhende individuelle Präferenzstruktur unterstellt (vgl. u. a. Sears u. Funk 1999; bzgl. wohlfahrtstaatlicher Einstellungen siehe auch Andreß et al. 2001, S. 45-49; Wilkoszewski 2008). Die Annahmen decken sich dabei insofern mit denen des Alterseffekts, als dass der Self-Interest-Ansatz ebenfalls impliziert, dass Einstellungen lebenszyklischen Veränderungen unterliegen. Demnach sollten Ältere und Jüngere in erster Linie diejenigen Politiken als subjektiv relevant erachten, die für sie rentabel sind, das heißt von denen sie als Leistungsempfänger profitieren (werden). So ist anzunehmen, dass Ruheständler, die in der Regel in hohem Maße auf sozialstaatliche Transferleistungen angewiesen sind, ein besonderes Interesse an der Erhöhung gesetzlicher Rentenzahlungen haben und der Bereich der finanziellen Alterssicherung für sie daher eine besondere Bedeutung besitzt. Gleichzeitig sollten sie dem Bildungs- und Familiensektor eine geringere Relevanz zuschreiben, weil sie von diesen Leistungen naturgemäß selber nicht mehr profitieren werden. ${ }^{15}$ Umgekehrt sollten für Jüngere Bereiche wie die finanzielle oder pflegerische Absicherung zumindest aktuell weniger wichtig sein, da sie selber (noch) keine Leistungen aus diesen umlagefinanzierten Systemen erhalten. Für sie sind der Bildungs- und Familienbereich (vor allem wenn sie Kinder haben oder planen, eine Familie zu gründen) daher aller Wahrscheinlichkeit nach zum aktuellen Zeitpunkt relevanter. Da sie allerdings darauf hoffen, von sozialstaatlichen Leistungen zu profitieren, wenn sie das Rentenalter erreicht haben,

13 So zeigen empirische Untersuchungen, dass junge Grünen-Wähler der ersten Stunde ihre postmateriellen Einstellungen auch mit zunehmendem Alter beibehalten und der Partei größtenteils treu bleiben. Kroh und Schupp (2011, S. 7) sehen demzufolge die gesellschaftliche Alterung langfristig als einen „strukturellen Vorteil für die Grünen“ (vgl. hierzu auch Kohler 1998).

14 Da die RWS 1994 und 1998 nicht durchgeführt wurde, beruhen die Angaben für diese Jahre auf eigenen Berechnungen, die auf Basis von exit polls vorgenommen wurden.

15 Wilkoszewski (2008, S. 200) findet in seiner Analyse für Deutschland Hinweise auf einen derartigen Effekt in Bezug auf die Erhöhung des Kindergeldes: „[...] with every year gained in life, the odds to support a significant increase in child benefits decrease by $3.9 \%$, holding all other variables constant." 
sind diese Bereiche aus ihrer Sicht sicherlich nicht unbedeutend. Aufgrund der an das Lebensalter geknüpften wechselnden Wahrscheinlichkeiten, von bestimmten Leistungen zu profitieren, ist daher zu erwarten, dass in Politikfeldern, in denen Transfers ausschließlich auf Junge beschränkt sind, größere Einstellungsdifferenzen zwischen Jungen und Alten hinsichtlich der Wichtigkeit aber auch der gewünschten Ausgaben in diesen Bereichen auftreten als in Politikfeldern, die auf die Sicherstellung der Versorgung der älteren Bevölkerung abzielen (vgl. Bonoli u. Häusermann 2009, S. 215).

Einschränkend ist hinzuzufügen, dass diese Sichtweise die Integration des Individuums in generationenübergreifende soziale Netzwerke - insbesondere in die Familie - gänzlich unberücksichtigt lässt. Die familiären Beziehungen werden in verschiedenen Studien von dem Großteil der Befragten als sehr gut bewertet. Es finden wechselseitige intergenerationelle Transfers materieller und nicht-materieller Art statt, die vor allem den jeweiligen Bedürftigen im Familiengefüge zu Gute kommen (Blome et al. 2008, S. 33-35; Kohli 2009). Es ist daher verständlich und wenig überraschend, dass Ältere im Allgemeinen ein starkes Interesse am Wohlergehen ihrer Nachkommen haben und diesen bestmögliche Ausgangsbedingungen wünschen. Die Dominanz rein eigennütziger Motive in ihren Präferenzen erscheint folglich wenig plausibel. Vielmehr sollten auch das Bildungswesen und die Familienpolitik mit Blick auf davon profitierende Familienmitglieder eine gewisse Relevanz für sie haben. ${ }^{16}$ Unter diesem Blickwinkel erscheint die Ausweitung des Self-Interest-Ansatzes durch Einbeziehung des familiären Hintergrunds sinnvoll und notwendig.

Empirische Untersuchungen weisen zudem in der Tat darauf hin, dass Kinderlose und Eltern sich im Hinblick auf die geforderte Belastung beziehungsweise Unterstützung Jüngerer deutlich voneinander unterscheiden (Wilkozewski 2010, S. 377381). Hinsichtlich des in den letzten Dekaden kontinuierlich gestiegenen Anteils Kinderloser ${ }^{17}$ kann die Vermutung nicht von der Hand gewiesen werden, dass dieser festzustellende Zusammenhang zukünftig womöglich zu erhöhtem Widerstand der Älteren gegen eine politische Fokussierung auf die Bereiche Familie und Bildung führt, da kinderlose Ältere aufgrund ihrer Nichtbetroffenheit eine weitaus geringere Notwendigkeit für die Unterstützung dieser Politikbereiche sehen dürften.

Aus Sicht der Jungen erscheint es ebenso plausibel, dass sie ihre (Groß-)Eltern versorgt und abgesichert wissen möchten. Sie dürften daher kaum für eine Schlechterstellung der Rentner plädieren. ${ }^{18}$ Zudem wollen sie ebenfalls, trotz der

16 Unter Verweis auf den sogenannten sozialen Altruismus formuliert Wilkoszewski (2010) die These, dass sich auch (Groß-)Eltern, deren Nachkommen nicht mehr direkt von Zahlungen im Bildungs- oder Familienbereich profitieren, allein aufgrund der Erfahrung, ein Kind großgezogen zu haben, für Leistungserhöhungen in diesen Bereichen aussprechen.

17 Berechnungen des Statistischen Bundesamtes (2012, S. 28-29) zufolge ist etwa jede zehnte Frau der Geburtsjahrgänge 1933-1938 kinderlos, von den 1964 bis 1968 Geborenen trifft dies auf fast jede fünfte zu.

18 Nebeneffekte dieser Absicherung der Älteren durch sozialstaatliche Leistungen für die Jungen sind zum einen eine Entlastung von Verpflichtungen gegenüber ihren älteren Verwandten (vgl. Blome et al. 2008) und zum anderen die Möglichkeit, direkt finanziell profitieren zu können, denn finanzielle familiäre Transfers führen in den meisten Fällen von den Alten zu den Jungen; ohne Rentenzahlungen wären diese Transfers in vielen Fällen nicht möglich (vgl. u. a. Künemund 2002). 
zu erwartenden geringeren Rendite im Vergleich zu den Älteren, soweit möglich von gesetzlichen Rentenzahlungen profitieren. In diese Richtung deuten Befunde empirischer Studien, die eine ungebrochen hohe Akzeptanz der Rente in allen Altersgruppen feststellen (vgl. u. a. Dallinger 2002; Schrenker 2009). Dennoch kann auch in diesem Zusammenhang nicht übersehen werden, dass der steigende Anteil Kinderloser den Unwillen auf Seiten der Jüngeren wachsen lassen könnte.

Neben den bisher genannten Aspekten sind auch altersbedingt unterschiedliche Wahrnehmungen und Beurteilungen von zukünftigen Problemen, die sich durch die Alterung potenziell ergeben, zu erwarten. Angesichts des in der öffentlichen Diskussion vermittelten Eindrucks einer zunehmenden Funktionsunfähigkeit der sozialen Sicherungssysteme wird von einer pessimistischeren Einschätzung der zukünftigen Situation durch Jüngere ausgegangen. So sind die Beiträge zur gesetzlichen Rentenversicherung in den letzten Jahrzehnten gestiegen, während das durchschnittliche Rentenniveau rückläufig ist (vgl. Deutsche Rentenversicherung 2011, S. 238, 245). Diese gegenläufigen Entwicklungen verdeutlichen die negativen Konsequenzen der gesellschaftlichen Alterung für die abnehmende jüngere (Erwerbs-)Bevölkerung, welche die Renten von immer mehr Älteren zu finanzieren hat und daher in eine ungünstige Lage gerät. Damit geht einher, dass sie trotz gestiegener Beiträge zur gesetzlichen Rentenversicherung geringere Rentenzahlungen als ihre (Groß-)Eltern zu erwarten hat. Der dadurch entstehende Unmut könnte sich zukünftig ausweiten, sodass jüngere Erwerbstätige zunehmend unwilliger werden, in soziale Sicherungssysteme einzuzahlen (vgl. Blome et al. 2008, S. 316), zumindest aber mit größerer Skepsis in die Zukunft blicken.

Im Hinblick auf systematische Unterschiede im Stimmverhalten zwischen Älteren und Jüngeren sind altersspezifische Einstellungsdifferenzen, sei es bei der Einschätzung von Problemen oder der Wichtigkeit bestimmter damit verbundener Politikbereiche, allein jedoch nicht ausreichend. Es ist darüber hinaus notwendig, dass Wähler bei den Parteien unterschiedliche Positionen und Schwerpunktsetzungen wahrnehmen und diese auch mit ihren eigenen Präferenzen in Beziehung setzen beziehungsweise bewerten können (vgl. Campbell et al. 1980 [1960], S. 168-187). Sicherlich ist es abwegig, zu behaupten, dass es das politische Geschehen in Deutschland prägende Parteien gibt, die sich ausschließlich für die Interessen einer Altersgruppe einsetzen. Die Durchsicht von Parteiprogrammen wird kaum eine solch einseitige Ausrichtung erkennen lassen. Allerdings ist nicht zu bestreiten, dass Parteien unterschiedliche Profile haben und pflegen, die durchaus stärker oder schwächer mit den Interessen spezifischer Altersgruppen kompatibel sind (Wilkoszewski 2003, S. 90-122). Zieht man die altersstrukturelle Zusammensetzung der Parteien als Indiz einer altersspezifischen Interessenvertretung heran, so schneiden die Grünen 2009 mit einem Anteil von 15 Prozent unter 35-Jähriger in dieser Altersgruppe am besten ab. Die CDU kommt hier lediglich auf sieben Prozent. Dagegen sind knapp 45 Prozent der CDU-Mitglieder älter als 65 Jahre (bei der SPD liegt der entsprechende Anteil bei 37 Prozent), während dies nur auf zehn Prozent der Mitglieder von Bündnis 90/Die Grünen zutrifft (Klein 2011, S. 44-47). Diese deutlichen Unterschiede zwischen den Parteien sollten sich in einer zwischen Alt und Jung divergierenden Perzeption der Vertretung ihrer jeweiligen Anliegen sowie der zugewiesenen Lösungskompetenzen widerspiegeln. 
Für die empirische Überprüfung der dargestellten Überlegungen zu den politischen Folgen des demografischen Wandels sind zum einen die unterschiedlichen Wahlbeteiligungsraten einzelner Altersgruppen sowie zum anderen die zwischen diesen divergierenden Parteipräferenzen zentral. In Verbindung mit Verschiebungen in der Altersstruktur wirft dies die Frage nach den Konsequenzen für politische Einstellungen und die Wahlchancen der politischen Parteien auf. Wir nähern uns dieser Frage, indem wir zunächst untersuchen, ob bezüglich relevanter politischer Einstellungen und Präferenzen Differenzen zwischen jüngeren (18-35 Jahre) und älteren (65 Jahre und älter) Befragten bestehen. Die Ergebnisse dieser Analyse werden anschließend auf ihre Bedeutung hinsichtlich eines unterschiedlichen Stimmverhaltens zwischen Alt und Jung untersucht.

\section{Datenbasis}

Die folgenden empirischen Analysen basieren auf Daten einer Bevölkerungsumfrage, bei der von Januar bis September 2011 insgesamt 3911 volljährige Personen mit Wohnsitz in Deutschland telefonisch befragt wurden. Da ältere und jüngere Menschen in zufällig rekrutierten Umfragen aufgrund ihrer geringeren Teilnahmebereitschaft zumeist unterrepräsentiert sind, erfolgte ein disproportionales Oversampling dieser beiden Befragtengruppen. Gleichzeitig wurde der regional differenzierten demografischen Situation in Deutschland Rechnung getragen, indem ein regionalisiertes Oversampling von vergleichsweise schwach und vergleichsweise stark alternden Stadt- und Landkreisen durchgeführt wurde. ${ }^{19}$ Hierfür wurden in West- und Ostdeutschland anhand von Faktorenanalysen, die sowohl die aktuelle regionale Altersstruktur (statisches Kriterium) als auch deren Entwicklung seit 1994 (dynamisches Kriterium) berücksichtigen, diejenigen Kreise mit der extremsten Altersstruktur(-entwicklung) identifiziert. ${ }^{20}$ Die Zusammensetzung der Stichprobe ermöglicht somit eine dezidierte Untersuchung von politischen Einstellungen und Verhaltensweisen vor dem Hintergrund regionaler Altersstrukturunterschiede. Dies ist im deutschen Kontext von besonderer Relevanz, da diejenigen Regionen, die besonders stark vom demografischen Wandel betroffen sind, zu einem großen Teil in Ostdeutschland liegen. Um die starken Niveauunterschiede in der altersstrukturellen Zusammensetzung zwischen West- und Ostdeutschland angemessen zu berücksichtigen, werden die empirischen Analysen im folgenden Abschnitt daher für beide Landesteile getrennt durchgeführt.

19 Durch die Verwendung geeigneter Anpassungsgewichte ist sichergestellt, dass die empirischen Analysen jeweils repräsentativ für die gewählte Untersuchungspopulation sind. Für die Erstellung der Gewichte wurde auf Daten der statistischen Landesämter als Referenzsollwerte zurückgegriffen. Die Anpassungen erfolgten dabei für die folgenden Variablen(-kombinationen): Herkunftsregion (West-, Ostdeutschland [inkl. Berlin]; junge/alte Kreise), Geschlecht, formaler Schulabschluss (Hauptschule und niedriger, Realschule o. ä., Abitur), kategorisierte Altersgruppen (18-20, 21-30, 31-40, 41-50, 51-60, 61-70, 71-80, 80+), Bildung x Alter, Geschlecht $x$ Bildung sowie Geschlecht $x$ Alter.

20 Insgesamt wurden auf diese Weise 99 Kreise ermittelt - 28 (49) in jungen (alten) westdeutschen Regionen und 9 (13) in jungen (alten) ostdeutschen Regionen - wobei sich die Anzahl nach der Bevölkerungszahl richtete. Insgesamt leben jeweils etwa zehn Prozent der Bundesbevölkerung in den ,jungen“ und „,alten“ Kreisen. Berlin wurde bei der Berechnung ausgeklammert. 


\section{Empirische Analysen}

\subsection{Altersspezifische Einstellungsunterschiede}

Hinsichtlich der Wichtigkeit der verschiedenen Politikfelder (vgl. Tabelle O-1 im Online-Anhang ${ }^{21}$ ) ist zunächst anzumerken, dass sich die zugeschriebenen Bedeutungen in beiden Altersgruppen insgesamt auf sehr hohem Niveau bewegen. Unsere Erwartungen bezüglich altersspezifischer Unterschiede betreffend ist festzustellen, dass diese nur teilweise erfüllt werden. Es kann bestätigt werden, dass Älteren im Vergleich zu Jüngeren die finanzielle und pflegerische Altersabsicherung signifikant wichtiger sind. Allerdings gilt dies auch für die ,jüngeren “ Politikfelder, wobei die Differenzen in Ostdeutschland kein statistisch signifikantes Niveau erreichen.

Weiter oben wurde bereits darauf hingewiesen, dass die Annahme von rein an eigenen Interessen ausgerichteten Präferenzen angesichts der familiären Eingebundenheit zweifelhaft erscheint. Zur Ermittlung der Rolle intergenerationaler Bindungen im Bildungs- und Familienbereich untersuchen wir daher, ob die Angaben der Befragten unterschiedlich ausfallen, je nachdem, ob sie Kinder haben oder nicht. In diesem Punkt bestätigen die Ergebnisse unsere Vermutungen weitgehend. In Ostdeutschland ist festzustellen, dass beide Bereiche jüngeren Eltern etwas wichtiger sind. Umgekehrt schreiben Kinderlose unabhängig ihres Alters diesen Politikfeldern tendenziell weniger Bedeutung zu. ${ }^{22}$ In Westdeutschland zeigen sich gleich gelagerte nennenswerte Unterschiede lediglich in der Familienpolitik.

Bezüglich der Frage, welche Veränderungen hinsichtlich der Ausgaben in den Politikfeldern befürwortet werden, ist generell festzustellen, dass sich die Befragten in allen Bereichen für eine Erhöhung der staatlichen Ausgaben aussprechen (vgl. Tabelle O-2 im Online-Anhang). Allerdings fordern Jüngere fast durchgängig eine signifikant stärkere Anhebung als die Älteren. Lediglich in Ostdeutschland wünschen ältere Befragte in „alten“ Politikbereichen ein ähnlich hohes finanzielles Engagement des Staates wie jüngere. Analog zur Vorgehensweise bei der Wichtigkeit haben wir auch hier in den ,jungen “ Politikfeldern danach unterschieden, ob die Befragten kinderlos sind oder nicht. Im Bereich der Bildung zeigen sich dabei kaum Unterschiede. In der Familienpolitik sprechen sich jüngere Eltern in beiden Landesteilen hingegen erwartungskonform für eine stärkere Ausgabenerhöhung aus.

Es ist bereits angeklungen, dass Unsicherheiten in Bezug auf das eigene Wohlergehen im Alter unter Jüngeren verbreiteter sein sollten. In einem nächsten Schritt untersuchen wir daher zum einen, inwieweit die gesellschaftliche Alterung als problematisch wahrgenommen wird. Zum anderen betrachten wir altersspezifische Einstellungsunterschiede hinsichtlich der individuellen Befürchtung, im Alter unzureichend versorgt zu sein. Unsere Ergebnisse verdeutlichen, dass die Befragten hinsichtlich der Folgen des demografischen Wandels offenbar zwischen einer sozialen und einer personalen Betroffenheitsebene unterscheiden (vgl. Tabelle O-3

21 Der Online-Anhang zum Beitrag kann unter www.pvs.nomos.de abgerufen werden.

22 Aufgrund der niedrigen Fallzahl älterer Kinderloser in Ostdeutschland sind die Ergebnisse allerdings mit Vorsicht zu interpretieren. 
im Online-Anhang). Die bevorstehenden Probleme für Deutschland werden als erheblich stärker eingeschätzt als die individuellen Sorgen, altersspezifische Differenzen treten diesbezüglich nicht auf. Anders sieht es bei den subjektiven Sorgen aus: Jüngere Menschen bewerten ihre Zukunft im Hinblick auf eine Unterversorgung in finanzieller und medizinischer Sicht im eigenen Alter erwartungsgemäß signifikant pessimistischer als Ältere. Dies gilt in beiden Landesteilen, wobei die geäußerte Unsicherheit in Ostdeutschland grundsätzlich etwas stärker ausfällt als in Westdeutschland. Dies ist ein Hinweis darauf, dass sich die weiter vorangeschrittene Alterung in Ostdeutschland in den Einstellungen der Bevölkerung widerspiegelt. Die Bedeutung persönlicher Sorgen ist auch im Zusammenhang mit der eingeschätzten Absicherung im eigenen Alter zu sehen. Wie erwartet, sind besorgte Personen - unabhängig davon, welcher Altersgruppe sie angehören oder in welchem Landesteil sie leben - hinsichtlich ihrer Zukunft unsicherer. Die signifikanten Unterschiede zwischen den Altersgruppen bleiben dabei weitgehend erhalten.

Bis hierhin zeigen sich zum Teil deutliche Einstellungsunterschiede zwischen Jung und Alt. Uns interessiert nun, ob diese von den Wählern auch mit Parteien in Verbindung gebracht werden. Dazu untersuchen wir, inwieweit Jüngere und Ältere ein Ungleichgewicht in der Vertretung ihrer Anliegen durch die politischen Parteien wahrnehmen (vgl. Tabelle O-4 im Online-Anhang). ${ }^{23}$ Es wird deutlich, dass sich Jüngere in beiden Landesteilen signifikant besser durch die kleineren Parteien FDP, die Linke und insbesondere die Grünen vertreten fühlen. Demgegenüber sehen sie sich von der CDU/CSU schlechter vertreten als Ältere - allerdings ist der Unterschied nur in Westdeutschland signifikant. Bei der SPD existieren keine altersspezifischen Unterschiede. Unsere Ergebnisse illustrieren, dass Parteien von verschiedenen Altersgruppen hinsichtlich ihrer politischen Interessenvertretung differenziert wahrgenommen werden und bestätigen damit weitgehend unsere Vermutungen altersspezifischer Parteienprofile.

Schließlich betrachten wir, inwiefern sich Alt und Jung hinsichtlich der den Parteien zugeschriebenen Lösungskompetenzen unterscheiden (vgl. Tabelle O-5 im Online-Anhang). Signifikante altersspezifische Unterschiede finden sich durchgängig bei der Bewertung der Unionsparteien in Westdeutschland, die Ältere in allen Politikbereichen am häufigsten als kompetent erachten. Demgegenüber besitzen Ältere in Ostdeutschland keine ausgeprägte Affinität zur CDU/CSU, vielmehr muss sie sich hier die Favoritenrolle mit der SPD teilen. Zudem gilt, dass Ältere die Linke als vergleichsweise fähiger bei der Lösung von Problemen einstufen als ihre Altersgenossen in Westdeutschland. Dies lässt sich auch bei Jüngeren in Ostdeutschland feststellen, die der Linken zu Lasten der Grünen und insbesondere der SPD häufiger eine Problemlösungskompetenz bescheinigen. Eine mögliche Erklärung hierfür ist die Sondersituation im Verhältnis zwischen diesen beiden Parteien in Ostdeutschland, wo die Linke zumindest teilweise in Regierungsverantwortung steht. Dagegen sehen jüngere Westdeutsche die SPD in nahe-

23 Erhoben wurden diese Einschätzungen, indem für jede Partei gefragt wurde, inwieweit sie die Interessen der Jüngeren einerseits sowie der Älteren andererseits vertritt. Für die Analysen werden nur die mit der jeweiligen Altersgruppe korrespondierenden Antworten verwendet. 
zu allen Bereichen als die kompetenteste Partei an. ${ }^{24}$ Die Prozentsätze der Nennungen liegen allerdings deutlich unter dem jeweiligen Niveau der älteren Westdeutschen für die Unionsparteien. Darüber hinaus gibt es erwartungsgemäß altersspezifische Differenzen bei der Bewertung der Grünen, denen die jüngeren Westdeutschen eine höhere Kompetenz - insbesondere in den „jungen“ Politikfeldern - zusprechen. Schließlich ist festzustellen, dass die FDP hinsichtlich ihrer Lösungskompetenz insbesondere in Ostdeutschland von Älteren erheblich schlechter beurteilt wird als von Jüngeren.

Insgesamt zeigen diese Ergebnisse deutliche Einstellungsunterschiede zwischen Alt und Jung. Darüber hinaus werden Parteien von verschiedenen Altersgruppen sehr unterschiedlich wahrgenommen. Inwieweit dies Auswirkungen auf die Stimmabgabe hat, wird im folgenden Abschnitt untersucht. Würden die altersspezifischen Einstellungen tatsächlich $\mathrm{zu}$ einem systematisch unterschiedlichen Stimmverhalten führen, dann hätte der faktische Anstieg des Anteils älterer Wähler in der Tat potenziell weitreichende Konsequenzen für den Ausgang von (zukünftigen) Wahlen und die Entwicklung zu einer „Rentner-Demokratie“.

\subsection{Altersspezifische Determinanten der Wahl einer Partei}

Zur Überprüfung eines systematisch unterschiedlichen Wahlverhaltens zwischen Alt und Jung verwenden wir ein konditionales Logit-Modell zur Erklärung der Parteiwahl (vgl. u. a. Alvarez u. Nagler 1998). Auf diese Weise kann die Bedeutung der einbezogenen Erklärungsvariablen für die Wahlwahrscheinlichkeiten der einzelnen Parteien gemeinsam analysiert werden. Falls sich die Koeffizienten der Determinanten zwischen Älteren und Jüngeren signifikant unterscheiden, ist das ein deutlicher Hinweis auf ein divergierendes Stimmverhalten. Als Erklärungsvariablen dienen die für Deutschland aufgrund der gesellschaftlichen Alterung erwarteten Probleme, die, wie im vorherigen Abschnitt gezeigt wurde, ein beträchtliches Ausmaß aufweisen, sowie die Kompetenzzuweisungen an die Parteien in den schon mehrfach genannten Politikbereichen. Die Aufnahme der Stärke der Parteiidentifikation erfolgt in erster Linie als Kontrollvariable, um eine längerfristige affektive Bindung an eine Partei zu berücksichtigen. Die Zelleneinträge in Tabelle 1 enthalten die Logit-Koeffizienten ${ }^{25}$ für jüngere Befragte zwischen 18 und 35 Jahren sowie für ältere Befragte ab 65 Jahren. Zudem sind die uns besonders interessierenden Differenzen zwischen beiden Altersgruppen ausgewiesen. Auf die Darstellung separater Modelle für West- und Ostdeutschland wird verzichtet, da eine Differenzierung - anders als bei den Niveauunterschieden im vorigen Abschnitt - nicht zu inhaltlich anderen Schlussfolgerungen führt.

Gemessen an der Gesamterklärungskraft zeigt sich eine gute Modellanpassung. Dies weist auf deutliche Unterscheide zwischen den berücksichtigten Parteien hinsichtlich der aufgenommenen Erklärungsvariablen hin. Zudem indiziert die Diffe-

24 Der Bildungsbereich stellt diesbezüglich eine Ausnahme dar; hier sind die jeweiligen Anteile für die CDU/CSU und die SPD nahezu gleich.

25 Auf die Angabe von Standardfehlern wurde aus Gründen der Übersichtlichkeit verzichtet. Diese können aber, wie auch die komplette Stata-Syntax, bei den Autoren angefragt werden. 
renz in der Varianzaufklärung, die für Ältere höher ausfällt, signifikante Bedeutungsunterschiede zwischen den Altersgruppen hinsichtlich der verwendeten Einstellungsvariablen. Ein Blick auf die Konstanten verdeutlicht, dass sich Jung und Alt im Hinblick auf ihre Stimmabgabe grundsätzlich voneinander unterscheiden, wenn sämtliche Erklärungsvariablen den Wert Null annehmen. ${ }^{26}$ So bedeutet der signifikante positive Effekt bei den Jüngeren für die Grünen eine höhere vorhergesagte Wahlwahrscheinlichkeit gegenüber der CDU/CSU, während der negative Effekt für die Linke auf eine geringere Wahlwahrscheinlichkeit hinweist. Demgegenüber zeigen die durchgängig negativen Koeffizienten bei älteren Befragten eine geringere Wahlwahrscheinlichkeit aller Parteien im Vergleich zur CDU/ CSU an, was als Bestätigung unserer Ausführungen zu Beginn von Abschnitt 2 verstanden werden kann und auf grundsätzlich divergierende Parteiprädispositionen zwischen Alt und Jung hindeutet. Besonders deutlich wird dies im Fall der FDP, bei der die Differenz zwischen Älteren und Jüngeren mit Abstand am höchsten ausfällt.

\section{Tabelle 1: Bedingte logistische Regression zur Vorhersage der Wahlabsicht} (Fünf-Parteien Wahl)

\begin{tabular}{|c|c|c|c|}
\hline & $\begin{array}{c}\text { jüngere } \\
\text { Befragte } \\
(18-35)\end{array}$ & $\begin{array}{c}\text { ältere } \\
\text { Befragte } \\
(\geq 65)\end{array}$ & $\begin{array}{l}\text { Differenz } \\
\text { (alt-jung) }\end{array}$ \\
\hline \multicolumn{4}{|c|}{$\begin{array}{l}\text { Probleme für Deutschland durch gesell- } \\
\text { schaftliche Alterung (Referenz: CDU/CSU) }\end{array}$} \\
\hline SPD & $-0,29$ & $0,34^{+}$ & $0,63 *$ \\
\hline FDP & $-0,06$ & $1,45 * *$ & $1,51 * *$ \\
\hline Bündnis 90/Die Grünen & $-0,23$ & $0,47^{*}$ & $0,71 * *$ \\
\hline Die Linke & 0,26 & 0,13 & $-0,14$ \\
\hline \multicolumn{4}{|l|}{ Kompetenzzuweisungen an Parteien } \\
\hline Bildung (Kinderlose) & $1,08 * * *$ & $-1,29 *$ & $-2,37 * * *$ \\
\hline Bildung (Eltern) & $1,44 * * *$ & $0,68 * *$ & $-0,76^{+}$ \\
\hline Familie (Kinderlose) & $0,59 * * *$ & $-0,37$ & $-0,96^{+}$ \\
\hline Familie (Eltern) & $1,34 * * *$ & $0,95 * *$ & $-0,38$ \\
\hline finanzielle Absicherung im Alter & $0,81 * * *$ & $1,11 * * *$ & 0,30 \\
\hline Pflege im Alter & $0,54 * *$ & $0,65 * *$ & 0,11 \\
\hline \multicolumn{4}{|l|}{ Stärke der Parteiidentifikation } \\
\hline $\mathrm{CDU} / \mathrm{CSU}$ & $0,79 * * *$ & $1,72 * * *$ & $0,93 * * *$ \\
\hline SPD & $0,68 * * *$ & $0,60 * * *$ & $-0,07$ \\
\hline FDP & $0,19 * *$ & $0,33 * *$ & 0,14 \\
\hline Bündnis 90/Die Grünen & $0,88 * * *$ & $0,21 * * *$ & $-0,67 * * *$ \\
\hline Die Linke & $0,45 * * *$ & $0,70 * * *$ & $0,24^{+}$ \\
\hline
\end{tabular}

26 Das ist dann der Fall, wenn keine Partei für kompetent in den aufgeführten Bereichen erachtet wird und zudem eine mittlere Problemperzeption sowie eine mittlere Stärke der Parteineigung angenommen wird. 


\begin{tabular}{lccc}
\hline & $\begin{array}{c}\text { jüngere } \\
\text { Befragte } \\
(\mathbf{1 8 - 3 5 )}\end{array}$ & $\begin{array}{c}\text { ältere } \\
\text { Befragte } \\
(\geq \mathbf{6 5})\end{array}$ & $\begin{array}{c}\text { Differenz } \\
\text { (alt-jung) }\end{array}$ \\
\hline $\begin{array}{l}\text { Konstanten } \\
\text { (Referenz: CDU/CSU) }\end{array}$ & & & \\
$\quad$ SPD & 0,49 & $-0,54$ & $-1,03$ \\
$\quad$ FDP & $-0,81$ & $-5,58^{* * *}$ & $-4,78^{* *}$ \\
$\quad$ Bündnis 90/Die Grünen & $1,04^{*}$ & $-0,01$ & $-1,05$ \\
$\quad$ Die Linke & $-1,73 *$ & $-0,72$ & 1,01 \\
\hline N (Alternativen) & 3560 & 3890 & 7450 \\
N (Fälle) & 712 & 778 & 1490 \\
McFadden's Pseudo-R ${ }^{2}$ & 0,65 & 0,76 & $0,12 * * *$ \\
\hline
\end{tabular}

Signifikanzen: ${ }^{+}: p<0,10,{ }^{*}: p<0,05,{ }^{* *}: p<0,01,{ }^{* * *}: p<0,001 ;$ Wertebereich der Stärke der Parteiidentifikationen jeweils von o (keine) bis 1 (sehr stark); repräsentativ für Gesamtdeutschland gewichtet.

Bezüglich der Konsequenzen der erwarteten Probleme aufgrund der gesellschaftlichen Alterung für die Wahlabsicht fällt auf, dass bei fast allen Parteien signifikante Differenzen zwischen jüngeren und älteren Befragten bestehen. Dies bedeutet, dass sich eine Veränderung der Problemperzeption in den beiden Altersgruppen unterschiedlich auswirkt. Bei älteren Befragten sind die Koeffizienten durchgehend positiv und mit Ausnahme der Linken statistisch signifikant. Eine stärkere Problemwahrnehmung geht in diesem Fall mit einer höheren Wahrscheinlichkeit für die Wahl einer dieser Parteien gegenüber der CDU/CSU als Referenzgruppe einher. Dieses Ergebnis ist zunächst überraschend, allerdings muss hierbei berücksichtigt werden, dass sich das grundsätzliche Niveau der vorhergesagten Stimmanteile deutlich zwischen den Altersgruppen unterscheidet. Grafisch kann dies durch Conditional-Effect-Plots veranschaulicht werden (vgl. Abbildung O-1 im Online-Anhang). Diese verdeutlichen zunächst die Zunahme der Wahlwahrscheinlichkeit für die SPD, die FDP und die Grünen bei steigender Problemwahrnehmung (und gleichzeitiger Fixierung der übrigen Einflussfaktoren) unter älteren Befragten. Für sie liegt die vorhergesagte Wahrscheinlichkeit, etwa Bündnis 90/ Die Grünen zu wählen, jedoch unabhängig von den erwarteten Problemen aufgrund der gesellschaftlichen Alterung deutlich unter derjenigen jüngerer Wähler. Eine stärkere Problemwahrnehmung führt bei ersteren demnach zwar zu einer höheren Wahlwahrscheinlichkeit der Grünen, während der gegenteilige Effekt bei Jüngeren festzustellen ist, allerdings liegt der vorhergesagte Stimmanteil älterer Wähler selbst bei einer sehr starken Problemperzeption unter dem von jüngeren Wählern. Dagegen fällt die vorhergesagte Wahlwahrscheinlichkeit älterer Befragter für die CDU/CSU stets höher aus als die jüngerer Probanden.

Für den Einfluss der Zuweisung von Lösungskompetenzen an bestimmte Parteien durch die Wähler sind grundsätzlich positive Koeffizienten zu erwarten: Wird eine bestimmte Partei in einem der aufgeführten Politikbereiche als kompetent erachtet, sollte die Wahrscheinlichkeit, diese Partei auch zu wählen, signifikant höher sein. Aufgrund des festgestellten Einflusses von Kinderlosigkeit wurden zusätzlich zwei Interaktionsterme in das Modell aufgenommen, für die wir 
die Lösungskompetenz in den Bereichen Bildung und Familie jeweils mit der Elternschaft (ja/nein) der Befragten multiplizierten. Für die einzelnen Parteien ist ungefähr eine Verdopplung ihrer jeweils vorhergesagten Wahlwahrscheinlichkeit zu konstatieren, wenn sie im Bildungsbereich als kompetent erachtet werden. ${ }^{27}$ Der Einfluss der Kompetenzzuweisung ist für junge Eltern ebenfalls positiv, fällt aber stärker aus als bei Kinderlosen. Interessant sind nun die unterschiedlichen Effekte der Kompetenzzuweisung im Bereich Bildung für ältere Befragte. Hier ist der Haupteffekt sogar leicht negativ und kann dahingehend interpretiert werden, dass diese Determinante bei der Wahl einer Partei für kinderlose ältere Befragte nicht relevant ist. ${ }^{28}$ Haben die älteren Befragten dagegen Kinder, dreht sich der Einfluss um: Für sie ist die Kompetenz einer Partei im Bildungsbereich ein relevanter Prädiktor ihrer Wahlentscheidung, der die vorhergesagte Wahrscheinlichkeit wie bei Jüngeren deutlich erhöht.

Ein weitgehend identisches Muster zeigt sich für den Bereich Familie. Auch hier ist der Einfluss bei älteren Befragten ohne Kinder gänzlich anders als bei Älteren mit Kindern. Letztere weisen eine signifikant höhere vorhergesagte Wahrscheinlichkeit zur Wahl einer bestimmten Partei auf, wenn sie diese im Familienbereich als kompetent erachten. Insgesamt bestätigt sich somit die Erwartung, dass Politikfelder, in denen Ältere kein direktes eigenes Interesse mehr haben, für ihre Wahlentscheidung bedeutsam sind, sofern sie eigene Kinder haben. Intergenerationale Bindungen haben demzufolge auch für die Stimmabgabe eine nicht zu unterschätzende Bedeutung. Gleichzeitig lässt sich feststellen, dass eine Zuweisung von Lösungskompetenzen in Bereichen wie der finanziellen Altersabsicherung oder der Pflege im Alter auch bei Jüngeren einen positiven Effekt auf die vorhergesagte Wahlwahrscheinlichkeit ausübt.

Hinsichtlich der Stärke der Parteiidentifikation zeigen sich - wie nicht anders zu erwarten - signifikant positive Effekte für alle Parteien. Allerdings gibt es auch hier Differenzen zwischen beiden Altersgruppen: So deutet der positive Logit-Koeffizient für die altersspezifische Differenz in der Identifikationsstärke bei der CDU/CSU auf einen erheblich stärkeren Einfluss für ältere Wähler hin, während das gleiche mit umgekehrtem Vorzeichen für jüngere Wähler bei den Grünen zutrifft. Wie bereits weiter oben ausgeführt, gibt es altersspezifische Parteipräferenzen, die weitgehend unabhängig von den erläuterten Einstellungsunterschieden ein systematisch unterschiedliches Wahlverhalten zwischen Jung und Alt begründen.

Aus diesen Ausführungen wird somit zweierlei deutlich: Zum einen weicht das Stimmverhalten von Wählern über 64 Jahren zum Teil deutlich von dem jüngerer Wähler bis einschließlich 35 Jahren ab. Neben einer grundsätzlichen Prädisposition Jüngerer für die Grünen und Älterer für die CDU/CSU unterscheiden sich die

27 Eine Überprüfung dieses Effektes für alle fünf Parteien zeigte keine signifikanten parteienspezifischen Unterschiede, weshalb auf eine Differenzierung verzichtet wurde.

28 Dass dieser Effekt ein statistisch signifikantes Niveau erreicht, liegt in der Mittelung der Effekte über alle Parteien begründet und ist insofern teilweise ein Artefakt, da die Koeffizienten für die einzelnen Parteien für sich betrachtet keine statistische Signifikanz erreichen. Inhaltlich besitzen aber auch negative Koeffizienten eine gewisse Plausibilität. Etwa dann, wenn die Partei in den Augen der Wähler in diesem Bereich über keinerlei Kompetenz verfügt beziehungsweise ihre Kompetenzen in anderen Bereichen vermutet werden. 
Altersgruppen zum anderen auch im Hinblick auf Determinanten, die im Zusammenhang mit der gesellschaftlichen Alterung stehen. Besonders deutlich wird dies bei Politikbereichen, von denen ältere Wähler weder direkt über Transferzahlungen noch indirekt über intergenerationale Beziehungen betroffen sind. In dieser Personengruppe der älteren Kinderlosen spielen Themenfelder wie Bildung und Familie für die Wahlentscheidung nahezu keine Rolle. Umgekehrt gilt dies jedoch nicht für jüngere Wähler. Für sie stellen auch „alte“ Politikbereiche, von denen sie momentan noch nicht direkt profitieren, relevante Faktoren dar. Insgesamt besitzen diese altersspezifischen Differenzen allerdings einen vergleichsweise geringen Einfluss auf die Wahlabsicht einer Partei. In der Regel stellt die Wahlabsicht eine recht gute Annäherung an die tatsächliche Parteiwahl dar. Dennoch besteht die Möglichkeit, dass die weiter oben festgestellte divergierende Wahlbeteiligung zwischen Jung und Alt gegenüber den hier berichteten altersspezifischen Differenzen zu deutlicheren Unterschieden im Hinblick auf die letztliche Wahl einer bestimmten Partei führen könnte. Insofern implizieren unsere Ergebnisse eine eher konservative Schätzung des Einflusses altersspezifischer Unterschiede.

\section{Schlussfolgerungen}

Wir haben in diesem Beitrag politische Einstellungen und Verhaltensweisen vor dem Hintergrund des demografischen Wandels einer Analyse unterzogen. Angesichts der verwendeten querschnittlichen Datenbasis lag unser Fokus dabei nicht auf der Separierung von Alters- und Kohorteneffekten - dies ermöglichte erst eine längsschnittliche Betrachtung. Hierfür wurde auf andere Arbeiten verwiesen, die ihrerseits einen wichtigen Beitrag zur Erforschung von politischen Konsequenzen gesellschaftlicher Alterungsprozesse leisten (vgl. z. B. Konzelmann et al. 2012). Die dort erarbeiteten Befunde deuten darauf hin, dass eine Kombination aus Alters- und Kohorteneffekten das Stimmverhalten in Deutschland beeinflusst. Unsere Ergebnisse zu individuellen altersspezifischen Einstellungen in ausgewählten Bereichen (deren Bedeutung parallel zur Alterung der Gesellschaft zunimmt) als Determinanten eines divergierenden Wahlverhaltens zwischen Alt und Jung stellen eine Ergänzung zu diesen Untersuchungen dar und erlauben die Betrachtung bisheriger Analysen aus einem erweiterten Blickwinkel.

Unsere zentralen Befunde lassen sich wie folgt zusammenfassen: Jüngere sind im Hinblick auf ihre eigene Absicherung im Alter erwartungsgemäß wesentlich besorgter als Ältere. Die Politikbereiche, welche die Versorgung der älteren Bevölkerung sicherstellen, sind für sie wichtig, aber (noch) nicht ganz so zentral wie für Ältere. Jüngere plädieren zudem in allen abgefragten Politikbereichen für eine Erhöhung der staatlichen Mittel und unterscheiden sich darin signifikant von den Älteren. Entscheidend ist es, ob die Befragten Kinder haben oder nicht: Insbesondere die Familienpolitik hat für junge Eltern einen höheren Stellenwert im Vergleich zu Kinderlosen. Weiter haben wir festgestellt, dass sich - wie vermutet unterschiedliche Parteiprofile erkennen lassen. Jüngere sehen ihre politischen Interessen am besten durch die Grünen vertreten, Ältere wiederum nennen häufiger die Unionsparteien als Vertreter ihrer Anliegen. Dieses Ergebnis bestätigt sich hinsichtlich der Kompetenzzuschreibungen. 
Darüber hinaus haben wir überprüft, ob und inwiefern diese Einstellungsdifferenzen zu einem systematisch unterschiedlichen Stimmverhalten zwischen Alt und Jung führen. Denn erst wenn sich die Determinanten der Wahlabsicht zwischen den Altersgruppen unterscheiden, sind Befürchtungen einer „Rentner-Demokratie“ als Folge der gesellschaftlichen Alterung gerechtfertigt. Die Ergebnisse im vorangegangenen Abschnitt haben hierzu einige wichtige Befunde erbracht. So ist festzuhalten, dass ältere Wähler überproportional häufig der CDU/CSU ihre Stimme geben, während jüngere die Grünen und die SPD präferieren. Dies ist seit längerem bekannt und insofern lediglich eine Bestätigung bisheriger Erkenntnisse. Unsere Ergebnisse ergänzen die bestehenden Forschungsarbeiten jedoch um einen weiteren Aspekt: Einstellungen in Bereichen, die in besonderer Weise vom demografischen Wandel betroffen sind, tragen in nicht unerheblicher Weise zur Erklärung des altersspezifischen, jedoch nicht unbedingt parteienspezifischen, Stimmverhaltens bei. Differenzen in diesen Einstellungen beruhen dabei zu einem großen Teil auf Interessenlagen und Bedürfnissen, die sich im Laufe des Lebens verändern. In diese Richtung deutet etwa der geringere Einfluss von „jungen“ Politikbereichen im Hinblick auf die Wahlabsicht älterer Wähler. Gleichwohl ist dieser Befund nicht mit einer Entfremdung zwischen den Generationen gleichzusetzen. Vielmehr zeigen unsere Analysen, dass intergenerationale Beziehungen auch im Hinblick auf die Stimmabgabe eine entscheidende Rolle spielen und dadurch bewirken, dass Politikbereiche, die für Ältere keine direkte Relevanz mehr besitzen, aufgrund familiärer Bindungen dennoch das eigene Wahlverhalten beeinflussen.

Anhand dieser Erkenntnisse spricht wenig dafür, dass Jung und Alt diametral entgegengesetzte Interessenlagen haben, die ein innerhalb der Altersgruppen homogenes und zwischen den Altersgruppen divergierendes Wahlverhalten begründen. Dies spricht gegen die Etablierung von „grey majorities“ (Goerres 2008), die notwendige Reformen erschweren könnten. Was allerdings auffällt ist die Tatsache, dass die Elternschaft sowohl unter Jüngeren als auch unter Älteren offenbar einen konditionalen Einfluss auf die Ausrichtung von Interessen ausübt. Insofern kann vermutet werden, dass womöglich weniger rein altersspezifische Interessenlagen, sondern altersspezifische Interessenlagen in Kombination mit Elternschaft die Parteiwahl in einem gewissen Maße beeinflussen. Doch auch hier kann unserer Meinung nach zumindest aktuell noch nicht von einer das Stimmverhalten maßgeblich prägenden Lagerbildung zwischen Kinderlosen und Eltern gesprochen werden. Dem ist hinzuzufügen, dass Wähler bei ihrer Stimmabgabe stets auch die Angebotsseite der Politik berücksichtigen. Hier gibt es zumindest Anzeichen, dass Parteien zum einen in einer Art „vorauseilende[m] Gehorsam “ (Deutscher Bundestag 2010, S. 237) versuchen, die Interessen älterer Wähler zu bedienen und zum anderen einschneidende Reformen in die Zukunft verschieben. Wie zu Beginn dargestellt kann diese Haltung im Hinblick auf eine kurzfristige Wiederwahl durchaus rational sein - auch wenn sie langfristig zu ungünstigeren Ausgangsbedingungen für jüngere Generationen führen dürfte. Unsere Ergebnisse deuten darauf hin, dass trotz altersspezifischer Differenzen in zentralen Politikbereichen diese für einzelne Parteien weder besondere Vor- noch systematische Nachteile bedeuten. Hierfür sind die parteispezifischen Unterschiede im Umgang mit den Folgen des demografischen Wandels schlicht zu gering. Allerdings scheint sich auch bei den politischen 
Vertretern nach und nach die Erkenntnis durchzusetzen, dass ein „weiter so“ im Hinblick auf die Folgen des demografischen Wandels keine Option darstellt. Dies zeigt etwa die Entscheidung für eine schrittweise Erhöhung des Renteneintrittsalters, welche zwar noch keine umfassende, langfristige Strategie darstellt, die Problematik aber zumindest nicht länger ignoriert. Demzufolge ist die heraufbeschworene Angst vor einer „Rentner-Demokratie“, in der Parteien allein die Interessen Älterer im Blick haben, zum jetzigen Zeitpunkt vermutlich übertrieben. Dass die (älteren) Wähler eine einseitige Ausrichtung auf die Interessen der Älteren auch nicht wünschen, belegt die seit Jahren unverändert marginale Bedeutung von Parteien, die sich genau dies auf die Fahnen geschrieben haben.

Für die zukünftige Entwicklung von politischen Einstellungen und Verhaltensweisen ist angesichts des wachsenden Anteils älterer Menschen und der damit verbundenen negativen Folgen für die sozialen Sicherungssysteme allerdings eine Zunahme von Interessengegensätzen zwischen Jung und Alt beziehungsweise Eltern und Kinderlosen nicht auszuschließen. Dabei ist zu berücksichtigen, dass es im Hinblick auf die einzelnen Komponenten, welche die Entwicklung des demografischen Wandels bestimmen, regional sehr unterschiedliche Entwicklungen gibt. So sind insbesondere weite Gebiete Ostdeutschlands bereits in größerem Ausmaß von der Überalterung betroffen, während es in Westdeutschland voraussichtlich etwas länger dauern wird, bis die Folgen altersstruktureller Bevölkerungsverschiebungen flächendeckend sichtbar werden. Gleichwohl sind auch dort politische Auswirkungen bereits feststellbar, wie die Debatte um die Berücksichtigung regionaler Unterschiede im Verhältnis von Wahlberechtigten zu Minderjährigen (und damit implizit eines demografischen Faktors) bei einem Neuzuschnitt der Wahlkreise zeigt (vgl. Bielicki u. Kerscher 2012). Insgesamt dürfte der demografische Wandel in der Perzeption der Bürger daher weiter an Bedeutung gewinnen - und mit ihm aller Voraussicht nach auch die Bedeutung hiermit zusammenhängender Einstellungen für ihr Wahlverhalten. Von einer „Rentner-Demokratie“ zu sprechen, erscheint uns angesichts unserer Erkenntnisse allerdings nicht angebracht.

\section{Literatur}

Alber, Jens. 1994. Soziale Integration und politische Repräsentation von Senioren. In 60 plus. Die wachsende Macht der Älteren, Hrsg. Günter Verheugen, 145-168. Köln: Bundverlag.

Alvarez, R. Michael, und Jonathan Nagler. 1998. When Politics and Models Collide: Estimating Models of Multiparty Elections. American Journal of Political Science 42:55-96.

Andreß, Hans-Jürgen, Thorsten Heien, und Dirk Hofäcker. 2001. Wozu brauchen wir noch den Sozialstaat? Der deutsche Sozialstaat im Urteil seiner Bürger. Opladen: Westdeutscher Verlag.

Arzheimer, Kai. 2006. Jung, dynamisch, Nichtwähler? Der Einfluss von Lebensalter und Kohortenzugehörigkeit auf die Wahlbereitschaft. In Jugend und Politik: „Voll normal!”. Der Beitrag der politischen Soziologie zur Jugendforschung, Hrsg. Edeltraut 
Roller, Frank Brettschneider und Jan W. van Deth, 317-335. Wiesbaden: VS Verlag für Sozialwissenschaften.

Bielicki, Jan, und Helmut Kerscher. 2012. Karlsruhe lässt Wahlkreise neu zuschneiden. Süddeutsche Zeitung (23.02.2012), S. 6.

Blais, André, Elisabeth Gidengil, Neil Nevitte und Richard Nadeau. 2004. Where Does Turnout Decline Come from? European Journal of Political Research 43:221-236.

Blome, Agnes, Wolfgang Keck und Jens Alber. 2008. Generationenbeziehungen im Wohlfahrtsstaat. Lebensbedingungen und Einstellungen von Altersgruppen im internationalen Vergleich. Wiesbaden: VS Verlag für Sozialwissenschaften.

Bonoli, Giuliano, und Silja Häusermann. 2009. Who Wants what from the Welfare State? Socio-structural Cleavages in Distributional Politics: Evidence from Swiss Referendum Votes. European Societies 11:211-232.

Bundesministerium des Innern. 2011. Demografiebericht. Bericht der Bundesregierung zur demografischen Lage und künftigen Entwicklung des Landes.

Bundesministerium des Innern 2012. Jedes Alter zählt. Demografiestrategie der Bundesregierung.

Campbell, Angus, Philip E. Converse, Warren E. Miller und Donald E. Stokes. 1980 [1960]. The American Voter. Unabridged Edition. Chicago/London: University of Chicago Press.

Dallinger, Ursula. 2002. Die Akzeptanz der Rentenversicherung - gibt es einen Generationenkonflikt? Zeitschrift für Sozialreform 48:659-685.

Dalton, Russel J. 2008. The Good Citizen: How a Younger Generation is Reshaping American Politics. Washington: CQ Press.

Deutsche Rentenversicherung. 2011. Rentenversicherung in Zeitreihen. DRV-Schrift 22.

Deutscher Bundestag. 2010. Sechster Bericht zur Lage der älteren Generation in der Bundesrepublik Deutschland - Altersbilder in der Gesellschaft, BT-Drs. 17/3815. Berlin.

Dorbritz, Jürgen. 2008. Demographic Knowledge and Evaluation of Demographic Trends. In People, Population Change and Policies. Lessons from the Population Policy Acceptance Study, Vol. 2: Demographic Knowledge - Gender - Ageing, Hrsg. Charlotte Höhn, Dragana Avramov und Irena E. Kotowska, 21-44. Dordrecht: Springer Verlag.

Falter, Jürgen W., Uwe W. Gehring. 1998. Alter - ein neues Cleavage? In Wahlen und Wähler. Analysen aus Anlass der Bundestagswahl 1994, Hrsg. Hans-Dieter Klingemann und Max Kaase, 463-503. Opladen: Westdeutscher Verlag.

Goerres, Achim. 2008. Reforming the Welfare State in Times of Grey Majorities. The Myth of an Opposition between Younger and Older Voters in Germany. German Policy Studies 4:131-156.

Goerres, Achim. 2010. Das Wahlverhalten älterer Menschen. Forschungsergebnisse aus etablierten Demokratien. Zeitschrift für Parlamentsfragen 41:102-120.

Hagenaars, Jacques A. 1990. Categorical Longitudinal Data. Log-Linear Panel, Trend, and Cohort Analysis. Newbury Park u. a.: Sage.

Jennings, M. Kent, Richard G. Niemi. 1981. Generations and Politics. A Panel Study of Young Adults and their Parents. Princeton: Princeton University Press.

Kaspar, Hanna, und Jürgen W. Falter. 2007. Entstehung neuer Konfliktlinien: Geschlechterkonflikt und Alterskonflikt? In Der gesamtdeutsche Wähler. Stabilität und Wandel des Wählerverhaltens im wiedervereinigten Deutschland, Hrsg. Hans Rattinger, Oscar W. Gabriel und Jürgen W. Falter, 113-140. Baden-Baden: Nomos. 
Klein, Markus. 2011. Wie sind die Parteien gesellschaftlich verwurzelt? In Parteimitglieder in Deutschland, Hrsg. Tim Spier, Markus Klein, Ulrich von Alemann, Hanna Hoffmann, Annika Laux, Alexandra Nonnenmacher und Katharina Rohrbach, 39-59. Wiesbaden: VS Verlag für Sozialwissenschaften.

Klingholz, Reiner. 2012. Jedes Alter zahlt. Frankfurter Allgemeine Zeitung (25.04.2012), S. 25.

Kohler, Ulrich. 1998. Zur Attraktivität der Grünen bei älteren Wählern. Kölner Zeitschrift für Soziologie und Sozialpsychologie 50:536-559.

Kohli, Martin. 2009. Familiale Generationenbeziehungen im Wohlfahrtsstaat. In Altern in Deutschland. Altern: Familie, Zivilgesellschaft und Politik (Altern in Deutschland, Band 8), Hrsg. Jürgen Kocka, Martin Kohli und Wolfgang Streeck, 91-113. Stuttgart: Wissenschaftliche Verlagsgesellschaft.

Konzelmann, Laura, Corina Wagner und Hans Rattinger. 2012. Turnout in Germany in the Course of Time: Life Cycle and Cohort Effects on Electoral Turnout from 1953 to 2049. Electoral Studies 31:250-261.

Kroh, Martin, und Jürgen Schupp. 2011. Bündnis 90/Die Grünen auf dem Weg zur Volkspartei? Wochenbericht des DIW 78:2-9.

Künemund, Harald. 2002. Sozialstaatliche Leistungen und Familienbeziehungen im Alter - Verdrängung oder Ergänzung? In Zukunft der Soziologie des Alter(n)s, Reihe Alter(n) und Gesellschaft. Band 8, Hrsg. Gertrud M. Backes und Wolfgang Clemens, 167-181. Opladen: Leske + Budrich.

Miegel, Meinhard. 1981. Sicherheit im Alter: Plädoyer für die Weiterentwicklung des Rentensystems. Stuttgart: Verlag Bonn Aktuell.

Rattinger, Hans. 1992. Demography and Federal Elections in Germany, 1953-1990 - and beyond. Electoral Studies 11:223-247.

Rinne, Karin, Gert Wagner. 1995. Droht ein „Krieg der Generationen“? Empirische Evidenz zur Zufriedenheit mit der sozialen Sicherung. Sozialer Fortschritt 44:288-295.

Schrenker, Markus. 2009. Warum fast alle das deutsche Rentensystem ungerecht finden, aber trotzdem nichts daran ändern möchten. Die Wahrnehmung gerechter Renten und die Akzeptanz von Rentenreformen. Kölner Zeitschrift für Soziologie und Sozialpsychologie 61:259-282.

Sears, David O., und Carolyn L. Funk. 1999. Evidence of the Long-Term Persistence of Adults' Political Predispositions. The Journal of Politics 61:1-28.

Statistisches Bundesamt. 2012. Geburten in Deutschland. Wiesbaden: Statistisches Bundesamt.

Wilkoszewski, Harald. 2003. Die verdrängte Generation. Politische Parteien und die alternde Gesellschaft in Deutschland. Marburg: Tectum.

Wilkoszewski, Harald. 2008. Demographic Pressure and Attitudes towards Public Intergenerational Transfers in Germany. In Demographic Change and Intergenerational Justice, Hrsg. Joerg Chet Tremmel, 175-205. Berlin: Springer.

Wilkoszewski, Harald. 2010. Alte versus Junge. In Deutsche Kontraste 1990-2010: PolitikWirtschaft-Gesellschaft, Hrsg. Manuela Glaab, 355-385. Frankfurt a. M.: Campus Verlag.

Wolf, Jürgen, und Martin Kohli. 1998. Die politische Macht der Älteren und der Generationenkonflikt. In Altern und Gesellschaft. Gesellschaftliche Modernisierung durch Altersstrukturwandel, Hrsg. Wolfgang Clemens und Gertrud M. Backes, 147-169. Opladen: Leske + Budrich. 


\section{AutorInneninformationen:}

Dipl. Pol. Michael Bergmann,

Lehrstuhl für Vergleichende Politische Verhaltensforschung, Universität Mannheim, A5, 6, D-68131 Mannheim, michael.bergmann@uni-mannheim.de

[Korrespondenzautor]

Laura Konzelmann, M.A.,

Lehrstuhl für Vergleichende Politische Verhaltensforschung/Mannheimer Zentrum für Europäische Sozialforschung, Universität Mannheim, A5, 6, D-68131 Mannheim, laura.konzelmann@mzes.uni-mannheim.de

Prof. Dr. Hans Rattinger,

Lehrstuhl für Vergleichende Politische Verhaltensforschung, Universität Mannheim, A5, 6, D-68131 Mannheim, hans.rattinger@uni-mannheim.de 